\title{
Option pricing based on mixtures of distributions: Evidence from the Eurex index and interest rate futures options market
}

\author{
Sascha Wilkens \\ E-mail: Wilkens@gmx.de
}

Received: 10th February, 2005

\begin{abstract}
Sascha Wilkens works in financial risk advisory for an international consulting company. He holds a Doctorate/PhD in Finance from the University of Münster, Germany, and a Master's degree in Business Mathematics from the University of Hamburg, Germany. His research concentrates on empirical market studies and the pricing of derivative securities, including energy, weather and macroeconomic derivatives as well as structured financial products.
\end{abstract}

\section{Practical applications}

This paper considers several alternatives to the standard Black and Scholes assumption of $\log$-normally distributed security prices in an option pricing framework. Judging from empirical results for a comprehensive database of options transactions, a mixture of two or three log-normal kernels is found to perform well for price forecasting purposes, while the numerical implementation and calibration remain feasible. For non-path-dependent options, the technique could therefore serve as an alternative to complex process assumptions for the underlying, competing distributional shapes or other pricing approaches such as a direct fitting of smile curves.

\section{Abstract}

The use of flexible distributional assumptions in an option pricing framework has been analysed extensively in recent years. Based on a large set of transaction data from 1999 and 2000, this paper readdresses the suitability of flexible distributions for forecasting and hedging purposes of DAX and Euro-Bund-Future options traded on the Eurex. Implied risk-neutral distributions (RND) are derived via the original Black and Scholes model, the series expansion approach of Corrado and $\mathrm{Su}$ and models based on mixtures of two and three log-normal distributions. While, especially for DAX options, the more complex RND prove superior to the log-normality assumption within the Black-Scholes model in terms of forecasting prices, delta-neutral hedge portfolios are best derived from simple Black-Scholes hedge ratios.

\section{INTRODUCTION}

In addition to the substantial body of theoretical research on the valuation of 
contingent claims, many empirical investigations on the pricing of derivatives have been undertaken in recent decades (see, for example, the comprehensive investigations by Bakshi et al. ${ }^{1}$ and Dumas et $a .^{2}{ }^{2}$ ). In spite of the large size and steady growth of worldwide turnover in derivative contracts, both at exchanges and over the counter (OTC), analyses have focused mainly on the US market. Studies of the European Exchange (Eurex), currently the world's largest futures exchange, are rare. Apart from a few model-free analyses (see Mittnik and Rieken ${ }^{3,4}$ ), most studies concentrate on model-specific phenomena such as volatility smile and term structure in the standard Black-Scholes ${ }^{5}$ model (see, for example, Eberlein et al., ${ }^{6}$ Tompkins, ${ }^{7}$ Hafner and Wallmeier, ${ }^{8}$ Brunner and Hafner, ${ }^{9}$ Fengler et al., ${ }^{10}$ Fengler and Wang $\left.^{11}\right)$. The literature, however, offers only a few comprehensive studies on price forecasting and hedging functionality applied to representative option databases. For the Deutsche Terminbörse, the predecessor of the Eurex, Rachev and Mittnik $^{12}$ study DAX options between February 1992 and September 1995 with up to 60 days to maturity on the basis of an array of models (eg Laplace, Student and inverse Gaussian distributions). Belledin and Schlag ${ }^{13}$ investigate several competing models with stochastic volatility, applied to a very limited set of DAX options traded on the Deutsche Terminbörse between July and December 1996.

The purpose of this study is to fill this conspicuous gap in the literature by undertaking a pioneering investigation of option models for price forecasting and hedging purposes of Eurex DAX and Euro-Bund-Future options, based on data from 1999 and 2000. From a methodological point of view, the main technique consists in extracting risk-neutral distributions (RND) from a given cross-sectional set of options transactions. This mapping of the market pricing mechanism abstracts from specific assumptions about the stochastic evolution of market parameters. As a 'natural' extension of the original Black-Scholes model, the paper focuses on models based on mixtures of two and three log-normal distributions for the underlying. The use of such mixtures (for an introduction, see, for example, McLachlan and Peel ${ }^{14}$ ) can be motivated by their outstanding suitability for approximation purposes. For example, using an increasing number of normal mixture components, any distribution can be approximated to an arbitrary degree of precision. Ritchey ${ }^{15}$ was among the first to employ a mixture of (normal) distributions in option pricing. Subsequent research, including that of Bahra, ${ }^{16-18}$ Melick and Thomas, ${ }^{19}$ Guo $^{20}$ Söderlind and Svensson, ${ }^{21}$ Gemmill and Saflekos, ${ }^{22}$ Schittenkopf and Dorffner, ${ }^{23} \mathrm{Kim}$ and $\mathrm{Kim}^{24}$ and, very recently, Rebonato and Cardoso, ${ }^{25}$ assumes that the density of the terminal distribution of the underlying is a linear combination of log-normal densities. (For a discussion of the implications of the mixture approach, for example, with respect to the calculation of moments and the structure of the volatility smile, see Brigo and Mercurio. ${ }^{26}$ ) An economic interpretation is the assumption of multiple alternative regimes. The main advantage of the mixture 
approach is its high flexibility, allowing the approximation of a large array of distributions - without becoming excessively vulnerable numerically owing to the 'semi-parametric' character. The smooth behaviour of the tails - which rapidly decline and therefore, for example, prevent excessive kurtosis - is advantageous.

Resulting valuation formulae are of closed-form, facilitating parameter estimation and covering the Black-Scholes model as a special case. For a competing benchmark, refer additionally to the Gram-Charlier series expansion approach proposed by Corrado and $\mathrm{Su}^{27}$

On average, the more complex RNDs prove superior to the log-normality assumption within the Black-Scholes model in terms of forecasting ability, especially for DAX options, whereas delta-neutral hedge portfolios are best set up using hedge ratios derived from the simple Black-Scholes formula.

The remainder of the paper is organised as follows. In the second section, valuation formulae are derived for options in a mixture-of-distributions framework. The third section briefly reviews the series expansion approach to option valuation which serves as an additional benchmark for the mixture models. The data for the empirical study are described in the fourth section. The fifth section is devoted to the presentation of the results with respect to calculated (in-sample) RNDs, the models' (out-of-sample) forecasting ability and suitability for hedging purposes. The final section summarises the main results and provides an outlook for further research.

\section{OPTION PRICING BASED ON MIXTURES OF DISTRIBUTIONS}

\section{Overview}

The value of a derivative security can be expressed as an expected discounted pay-off, whereby the expectation is taken with respect to an appropriate risk-neutral density $f_{t, T}^{\star}{ }^{28}$ For the value $c_{t}$ of a European call option with strike $K$ and maturity $T$ at time $t \leq T$, this result is formalised as

$$
\begin{aligned}
c_{t}(K) & =\mathrm{e}^{-r(T-t)} E^{*}\left(\max \left\{S_{T}-K, 0\right\}\right) \\
& =\mathrm{e}^{-r(T-t)} \int_{K}^{\infty}\left(S_{T}-K\right) f_{t, T}^{*}\left(S_{T}\right) \mathrm{d} S_{T}
\end{aligned}
$$

where $r$ denotes the appropriate domestic interest rate (continuously compounded); $S_{T}$ the random spot price at the option's maturity; and $E^{*}$ the risk-neutral expectation operator. Breeden and Litzenberger $^{29}$ demonstrate that, if the risk-neutral distribution is continuous, its discounted density is equal to the second derivative of the European call option price with respect to the strike price

$$
f_{t, T}^{*}\left(S_{T}\right)=\left.\mathrm{e}^{r(T-t)} \frac{\partial^{2} c_{t}(K)}{\partial K^{2}}\right|_{K=S_{T}}
$$

Boundary conditions $\partial c_{t}(K) / \partial K \geq-\mathrm{e}^{-r(T-t)}$ and $\lim _{K \rightarrow \infty} \partial c_{t}(K) / \partial K=0$ as well as convexity and differentiability of $c_{t}(K)$ are assumed. Based on the boundary restrictions, it is easily shown that $f_{t, T}^{*}\left(S_{T}\right)$ $\geq 0$ and $\int_{0}^{\infty} f_{t, T}^{*}\left(K=S_{T}\right) \mathrm{d} K=1$ holds; ie $f_{t, T}^{*}\left(S_{T}\right)$ is indeed a density function.

Notably, the martingale restriction holds for each case $E^{*}\left(S_{T}\right)=\int_{0}^{\infty} S_{T} f_{t, T}^{*}\left(S_{T}\right) \mathrm{d} S_{T}=$ $\mathrm{e}^{\mathrm{r}(T-t)} S_{T}$.

The RND can be entirely (re)constructed from given European option 
prices and, in a complete market, be uniquely identified. For practical purposes, direct use of relations (1) and (2) faces the problem that option prices are observable only at a few traded strike prices. The structure of the RND between strike prices inevitably requires further assumptions. In addition, the function resulting from (2) will probably lack the desired smoothness. Methods for constructing RND from European option prices can basically be classified as follows (for a slightly different typology see, for example, Bahra ${ }^{17}$ ):

(1) Assumption of a specific stochastic process driving the security price.

(2) Assumption of a specific functional form of the terminal distribution - a more general approach, because a single terminal distribution can result from many different stochastic processes.

(3) Non-parametric techniques that are data oriented and largely free of parametric assumptions; Jackwerth ${ }^{30}$ distinguishes further between kernel methods, maximum entropy techniques and the group of approaches which directly fit implied volatilities and RND (methods of curve fitting).

Many models from categories (2) and (3) are reviewed by Jackwerth. ${ }^{30}$ Because of its flexibility and relative ease of implementation, this paper focuses on the class of parametric approaches from category (2) as a base technique and thereby especially on the mixture-of-distributions framework. Compared with non-parametric approaches, parametric methods generally are less vulnerable to overfitting and are useful as a means of ensuring a 'reasonable' distribution. ${ }^{21}$ Furthermore, parametric approaches contain submodels which facilitate an analysis of the required flexibility of the RND, while for non-parametric methods, there are no 'subcases'.

\section{Derivation of pricing formulae}

Within the Black-Scholes model, the assumption of a security process following a geometric Brownian motion results in an inflexible log-normal price distribution in any interval $[t, T]$. A (finite) mixture of $\mathrm{k}$ log-normal distributions can be seen as a 'natural' extension of the Black-Scholes framework. Modelling the (risk-neutral) security price distribution as a mixture of log-normal distributions accordingly leads to a mixture of normal distributions for the corresponding (risk-neutral) return distribution. This follows directly from the relationship between the density functions for return $\left(g_{t, T}^{*}\right)$ and security $\left(f_{t, T}^{*}\right)$ : $g_{t, T}^{*}(u)=S_{t} \mathrm{e}^{u} f_{t, T}^{*}\left(S_{t} \mathrm{e}^{u}\right)$.

Formally, let $k \in I N$

$$
\begin{aligned}
f_{t, T}^{\text {Mixture } k}\left(S_{T}\right) & =\sum_{j=1}^{k} \lambda_{j} f_{j}\left(\alpha_{j}, \beta_{i} ; S_{T}\right) \\
& =\sum_{j=1}^{\mathrm{k}} \frac{\lambda_{j}}{S_{T} \beta_{j}} \varphi\left(\frac{\ln S_{T}-\alpha_{j}}{\beta_{j}}\right), S_{T}>0
\end{aligned}
$$

with

$$
\sum_{j=1}^{k} \lambda_{j}=1, \quad \lambda_{j}>0 \forall j=1,2, \ldots, k
$$

and

$$
\begin{gathered}
\alpha_{j}=\ln S_{t}+\left(\varsigma_{j}-\frac{\sigma_{j}^{2}}{2}\right)(T-t), \\
\beta_{j}=\sigma_{j} \sqrt{T-t}
\end{gathered}
$$


$\varphi(x)$ stands for the density function of the standard normal distribution and $\lambda_{j}$ denotes the 'weight' of the $j$ th mixture component with parameters $\alpha_{j}$ and $\beta_{j}$, which themselves depend on (instantaneous) drifts $\boldsymbol{s}_{j}$ and volatilities $\sigma_{j}$ of the corresponding return distributions. It is obvious that (3) is indeed a density function with

$$
f_{t, T}^{\text {Mixture } k}\left(S_{T}\right) \geq 0 \forall S_{T}>0
$$

and

$$
\int_{0 t, T}^{\infty \text { Mixture } k}\left(S_{T}\right) \mathrm{d} S_{T}=1
$$

In order to exclude arbitrage opportunities in a complete market, an investment in the option underlying within each interval $[t, T]$ must yield the risk-free rate, ie $E^{*}\left(S_{T}\right)=S_{t} \mathrm{e}^{r(T-t)}$. With (3), this leads to the martingale constraint

$$
E^{*}\left(S_{T}\right)=\sum_{j=1}^{k} \lambda_{j} \exp \left(\alpha_{j}+\frac{\beta_{j}^{2}}{2}\right)=S_{t} \mathrm{e}^{r(T-t)}
$$

Without losing generality, the last drift parameter of the mixture, $\boldsymbol{s}_{k}, k \geq 2$ can be used to guarantee (6). Hence, for $\boldsymbol{s}_{k}$ it follows that

$$
\begin{aligned}
& \sum_{j=1}^{k} \lambda_{j} \mathrm{e}^{\mathrm{e}_{j}(T-t)}=\mathrm{e}^{r(T-t)} \Leftrightarrow \\
& \boldsymbol{S}_{k}=\frac{1}{T-t} \ln \left(\frac{\mathrm{e}^{r(T-t)}-\sum_{j=1}^{k-1} \lambda_{j} \mathrm{e}^{\mathrm{s}_{j}(T-t)}}{1-\sum_{j=1}^{k-1} \lambda_{j}}\right)
\end{aligned}
$$

Ensuring the existence of $\boldsymbol{s}_{k} \in I R$ requires

$$
\mathrm{e}^{r(T-t)}-\sum_{j=1}^{k-1} \lambda_{j} \mathrm{e}^{\boldsymbol{s}_{j}(T-t)}>0 \Leftrightarrow \sum_{j=1}^{k-1} \lambda_{j} e^{\left(\boldsymbol{s}_{j}-r\right)(T-t)}<1
$$

Based on the mixture of distributions, specific valuation formulae for European standard calls and puts, are then easily derived according to Cox and Ross ${ }^{28}$

$$
\begin{aligned}
c_{t}^{\text {Mixture } k}=\sum_{j=1}^{k} & \lambda_{j}\left[S_{t} \mathrm{e}^{\left(\mathbf{s}_{j}-r\right)(T-t)} \Phi\left(d_{j 1}\right)\right. \\
& \left.-K \mathrm{e}^{-r(T-t)} \Phi\left(d_{j 2}\right)\right] \\
p_{t}^{\text {Mixture } k=}=\sum_{j-1}^{k} & \lambda_{j}\left[K \mathrm{e}^{-r(T-t)} \Phi\left(-d_{j 2}\right)\right. \\
& \left.-S_{t} \mathrm{e}^{\left(\mathbf{s}_{j}-r\right)(T-t)} \Phi\left(-d_{j 1}\right)\right]
\end{aligned}
$$

with

$$
\begin{gathered}
d_{j 1}=\frac{\ln \left(\frac{S_{t}}{K}\right)+\left(s_{j}+\frac{1}{2} \sigma_{j}^{2}\right)(T-t)}{\sigma_{j} \sqrt{T-t}}, \\
d_{j 2}=d_{j 1}-\sigma_{j} \sqrt{T-t}
\end{gathered}
$$

$\Phi(x)$ as cumulative density function of the standard normal distribution and (6) to hold. Formulae (7) and (8) highlight that the mixture of densities leads to a linear combination of Black-Scholes expressions. Besides $S_{t}, K, r$ and $T-t$, the value of a European call or put in a model with $k$ mixture components depends on $k-1$ weights $\lambda_{j}, k-1$ parameters $\boldsymbol{s}_{j}$ and $k$ parameters $\sigma_{j}$, hence $3 k-2$ parameters in total. For $k=2$, and without explicitly demanding the martingale restriction to hold, Bahra ${ }^{17}$ provides a numerically advantageous transformation of these formulae.

\section{COMPETING BENCHMARK: MOMENT-ADJUSTED OPTION PRICING FORMULAE}

As an alternative to the Black-Scholes and mixture models, this paper additionally refers to the approach of Corrado and $\mathrm{Su},{ }^{27}$ which 
uses a Gram-Charlier series expansion of the (risk-neutral) return distribution, thus incorporating non-normal skewness $\left(\boldsymbol{\gamma}_{1}\right)$ and kurtosis $\left(\gamma_{2}\right)$ into option pricing. The value of a European call accordingly equals the Black-Scholes model value plus two correction terms that account for the higher moments of the return distribution

$$
c_{t}^{G C}=c_{t}^{B S}+\gamma_{1} \Theta_{3}+\left(\gamma_{2}-3\right) \Theta_{4}
$$

with

$$
\begin{aligned}
c_{t}^{B S}= & S_{t} \Phi\left(d_{1}\right)-K \mathrm{e}^{-r(T-t)} \Phi\left(d_{2}\right) \\
\Theta_{3}= & \frac{1}{3 !} S_{t} \sigma \sqrt{T-t}\left[\varphi\left(d_{1}\right)\left(2 \sigma \sqrt{T-t}-d_{1}\right)\right. \\
+ & \left.\sigma^{2}(T-t) \Phi\left(d_{1}\right)\right] \\
\Theta_{4}= & \frac{1}{4 !} S_{t} \sigma \sqrt{T-t}\left[\varphi\left(d_{1}\right)\right. \\
& \left(d_{1}^{2}-1-3 \sigma \sqrt{T-t}\right. \\
& \left.\left(d_{1}-\sigma \sqrt{T-t}\right)\right) \\
& \left.+\sigma^{3}(T-t)^{3 / 2} \Phi\left(d_{1}\right)\right]
\end{aligned}
$$

and

$$
\begin{gathered}
d_{1}=\frac{\ln \left(\frac{S_{t}}{K}\right)+\left(r+\frac{1}{2} \sigma^{2}\right)(T-t)}{\sigma \sqrt{T-t}}, \\
d_{2}=d_{1}-\sigma \sqrt{T-t}
\end{gathered}
$$

It is worth noting that in Corrado and $\mathrm{Su}^{27}$ (p. 179), there is a false sign in the expression for (3) in (12). Instead of a plus, the formula shows a minus sign (see also Brown and Robinson ${ }^{31}$ ). An analogous formula $p_{t}^{G C}$ for European put options can be derived by means of put-call parity. The direct derivation of a put formula, however, leads to an expression that, together with (10), does not fulfil put-call parity since, during the series expansion, terms of order five and higher are neglected.

One of the important constraints of the expansion model is that the implied (risk-neutral) return density $g_{t, T}^{*}(x)$ must fulfil $\int_{-\infty}^{\infty} g_{t, T}^{*}(x) \mathrm{d} x=1$ as well as $g_{t, T}^{*}(x) \geq 0$ for all $x$ from the support. The latter condition is necessary, since an approximation of a density function with the aid of a finite sum can also lead to negative function values. To guarantee that these conditions hold in (10), the parameters $\gamma_{1}$ and $\gamma_{2}$ must be restricted to certain intervals. Importantly, by means of series expansion, neither extremely skewed nor platykurtic return distributions can be captured (for more details see Jondeau and Rockinger $\left.^{32}\right)$.

\section{DATA}

The option products of the Eurex form the basis of the study. The analysis focuses on two popular products - index options on the German blue-chip stock index DAX (Deutscher Aktienindex) and options on the Euro-Bund-Future (Eurex ${ }^{33}$ ) — and their trades from January 1999 to December 2000. All option data were provided by Deutsche Börse, Frankfurt/Main, Germany.

DAX options are European-style and their underlying is the DAX performance index, based on the XETRA electronic trading system at the Frankfurt Stock Exchange. The index comprises the stocks of the 30 largest German exchange-listed companies. As a performance index, the valuation assumes a fictitious reinvestment of paid dividends into the index portfolio. Since the DAX performance index is also adjusted for any price-relevant corporate 
actions, it can be treated as a non-dividend paying asset. Euro-Bund-Future options are American-style and, as a special feature, are subject to a daily marking-to-market similar to their underlying (futures-style margining). The underlying future is based on fictitious notional long-term debt issued by the Federal Republic of Germany with a remaining time-to-maturity of between 8.5 and 10.5 years, and an interest coupon of six per cent per annum. Note that the underlying of Euro-Bund-Future options depends on their time to maturity: while the options can comprise maturities in any month, the Euro-Bund-Future expires on a quarterly basis. The options always refer to the next maturing Euro-Bund-Future. For example, options due in January, February and March are all based on the March Euro-Bund-Future contract.

When deriving RND from prices of American-style Euro-Bund-Future options, one does not need to control for the early exercise premium. For a futures-style option, it is never optimal to exercise it prematurely. ${ }^{34,35}$ Since Euro-Bund-Future contracts expire long before the bonds' specifics in the underlying (eg pull-to-par) become substantial, we do not have to account for the special interest rate-sensitive characteristics of the underlying.

For DAX options, the XETRA-DAX is used as input parameter for the underlying. The last trade price of the corresponding Euro-Bund-Future contract is assigned to the Euro-Bund-Future options. Intraday data for the XETRA-DAX were provided by Karlsruher Kapitalmarktdatenbank, Karlsruhe, Germany. Transactions data on the Euro-Bund-Future contracts were obtained from Deutsche Börse,

Frankfurt/Main, Germany. In the following, time-to-maturity is measured in calendar days and used in formulae as fractions of a year. Following standard practice, the appropriate term-dependent money market Euribor (European Interbank Offered Rate), available for intervals up to 12 months, is used as a proxy for the risk-free interest rate. Linearly interpolated rates are derived, while for times-to-maturity of more than one year, the 12-month Euribor is employed for simplicity.

In a preliminary step, the databases are corrected with respect to (1) insufficient time synchronisation between underlying and options market; and (2) options' times to maturity of less than seven calendar days or prices below ten ticks. Further cleaning steps refer to (3) the violation of distribution-free arbitrage boundaries; (4) the law-of-one-price; and (5) put-call parity. Since far in-the-money and out-of-the-money options are very rarely traded and therefore generally do not provide reliable and time-stable information, (6) moneyness $-M=\left(S_{t}-K\right) / K$ for calls and $M=\left(K-S_{t}\right) / K$ for puts - is restricted as follows: For DAX options, an interval $M \in[-0.25,0.25]$, for Euro-Bund-Future options $M \in[-0.05,0.05]$ is considered suitable. Note that no filtering with respect to implied volatility is applied, since this would imply a model-dependent cleaning that one explicitly wishes to avoid.

Table 1 provides an overview of characteristics of the cleaned databases, consisting of 1,195,820 records for DAX 
Table 1: Descriptive statistics for the cleaned data bases

$\begin{array}{llcccc}\text { Min. } & 10 & 25 & \text { Percentiles } & \\ & & 50 & 75 & 90 & \text { Max. }\end{array}$

\section{DAX options}

$\begin{array}{lrrrrrrr}\text { DAX index }^{\mathrm{a}} & 4,601.07 & 5,041.47 & 5,266.49 & 6,438.74 & 7,116.26 & 7,420.69 & 8,136.16 \\ \text { Strike }^{\mathrm{a}} & 3,750 & 4,900 & 5,300 & 6,200 & 7,100 & 7,600 & 10,400 \\ \text { Time-to-mat. }^{\mathrm{b}} & 7 & 10 & 16 & 29 & 50 & 100 & 732 \\ \text { Interest rate }^{\mathrm{c}} & 2.2824 & 2.6068 & 2.9040 & 3.3280 & 4.3780 & 4.8384 & 5.2736 \\ \text { Option price }^{\mathrm{a}} & 1.00 & 12.00 & 28.70 & 70.50 & 149.00 & 277.10 & 2,345.00 \\ \text { Moneyness } & -0.2500 & -0.1401 & -0.0852 & -0.0410 & -0.0102 & 0.0112 & 0.2499 \\ & & & & & & & \\ \text { II. Euro-Bund-Future }^{\text {options }} & & & & & & & \\ \text { Future }^{\mathrm{a}} & 101.88 & 103.49 & 104.86 & 105.80 & 109.36 & 113.85 & 116.33 \\ \text { Strike }^{\mathrm{a}} & 98.00 & 103.50 & 104.50 & 106.00 & 109.50 & 114.00 & 118.50 \\ \text { Time-to-mat. }^{\mathrm{b}} & 7 & 10 & 15 & 22 & 36 & 58 & 90 \\ \text { Option price }^{\mathrm{a}} & 0.10 & 0.13 & 0.20 & 0.35 & 0.60 & 0.95 & 5.67 \\ \text { Moneyness } & -0.0500 & -0.0190 & -0.0125 & -0.0066 & -0.0014 & 0.0018 & 0.0498\end{array}$

Notes: ${ }^{\mathrm{a}}$ points; ${ }^{\mathrm{b}}$ calendar days; ${ }^{\mathrm{c}} \%$ per annum (continuous compounding).

and 138,583 records for Euro-Bund-Future options, as well as their assigned market variables. Besides minimum and maximum, the respective 10-, 25-, 50-, 75- and 90-percentiles are displayed.

For both DAX and Euro-Bund-Future options, approximately one-third of the records in the cleaned databases belong to at-the-money (ATM) transactions; ie $M \in[-0.025,0.025]$ for DAX options and $M \in[-0.005,0.005]$ for Euro-Bund-Future options, while two-thirds are out-of-themoney or deep out-of-the-money trades. Notably, 55 per cent (70 per cent) of the DAX (Euro-Bund-Future) options records belong to very short-term options with seven to 30 calendar days to maturity.

\section{RESULTS}

\section{Implied risk-neutral distributions}

Given a set of $N_{1}\left(N_{2}\right)$ observed call (put) option prices $c_{t, T ; i}^{\text {Market }}\left(p_{t, T ; i}^{\text {Market }}\right)$, the $M$ model-implied parameters $\xi_{i}$ are obtained by minimising the sum of squared differences between these prices and the theoretical option values $c_{t, T ; i}^{\text {Model }}\left(p_{t, T ; i}^{\text {Model }}\right)$

$$
\begin{aligned}
& \arg \min _{\xi_{1}, \xi_{2}, \ldots, \xi_{M}} \sum_{i=1}^{N_{1}}\left(c_{t, T ; i}^{\text {Market }}-c_{t, T ; i}^{\text {Model }}\right)^{2} \\
& +\sum_{i=1}^{N_{2}}\left(p_{t, T ; i}^{\text {Market }}-p_{t, T ; i}^{\text {Model }}\right)^{2}
\end{aligned}
$$

The author refers to transactions prices because settlement prices are set by the 
Eurex and therefore do not always reflect actual trading opportunities. Based on the least-squares approach in (15), implied parameters for each interval $[t, T]$ are calculated for each model and trading day. Accordingly, all call and put options transactions enter the minimisation procedure (15). The data input, however, is restricted to those subsamples of options transactions with at least eight different strike prices in each estimation, so as to ensure reliable support for the extracted distributions. In order to investigate whether calibration for the Black-Scholes model (BS) should constrain on information from ATM options, the Black-Scholes model is also analysed by restricting the minimisation approach in (15) to ATM option trades (BS-ATM). The results are compared with those of the mixture models with two (Mixture-2) and three

(Mixture-3) kernels as well as with those of the Gram-Charlier model (GC).

The calculation of optimal parameter vectors according to (15) requires global optima of $m$-dimensional functions which consist of sums of highly non-linear terms. The usual optimisation and grid search algorithms tend to fail, especially for the mixture models with $m=4$ and $m=7$, respectively. Simulated annealing is therefore employed routinely for (stochastic) global optimisation (see, for example, Locatelli ${ }^{36}$ ), together with local optimisation algorithms (cf Rebonato and Cardoso ${ }^{25}$ for an alternative numerical approach to solving the optimisation problem posed in (15)). For the mixture models, the number of parameters that increase rapidly when adding new mixture components is a problem. Numerical stability is another issue which may become critical, especially for the higher moments of the distribution. ${ }^{37,38}$

For each model, there are a total of 2,630 parameter vectors for the DAX distributions and 657 parameter vectors for the distributions of the Euro-Bund-Future. Figure 1 depicts the implied estimations for the DAX option-implied RND on a randomly selected trading day, 19th November, 1999. The higher moments are particularly conspicuous. While the $\log$-normal security price distribution is always skewed to the right in the Black-Scholes model, skewness in all three alternative models is reversed to a right-steepness; the more pronounced the more flexible the model $(-0.41$ to -0.94$)$. A similar picture is obtained for kurtosis: the log-normal distribution with a value of approximately 3.0 faces the much more leptokurtic alternative distributions with values of up to 7.3. The figure also shows that for index values of approximately 5,300 or lower, the Black-Scholes alternatives have more probability mass in the left tail. For the right tails, such a phenomenon cannot be detected.

\section{Out-of-sample performance}

Based on the premise that estimated implied distributions are constant for short-term horizons, option prices on the following trading day are forecast (sliding windows technique). Therefore, all forecasts are run term-dependent.

For the DAX options, Table 2 provides the summary statistics for absolute and relative deviations in a one-day price 
Figure 1: Example of implicit risk-neutral security price distributions $\left(f_{t, T}^{*}\right)$ for DAX options. Sample data from 19th November, 1999; calculation from 2,247 options transactions; options' time-to-maturity was 28 calendar days

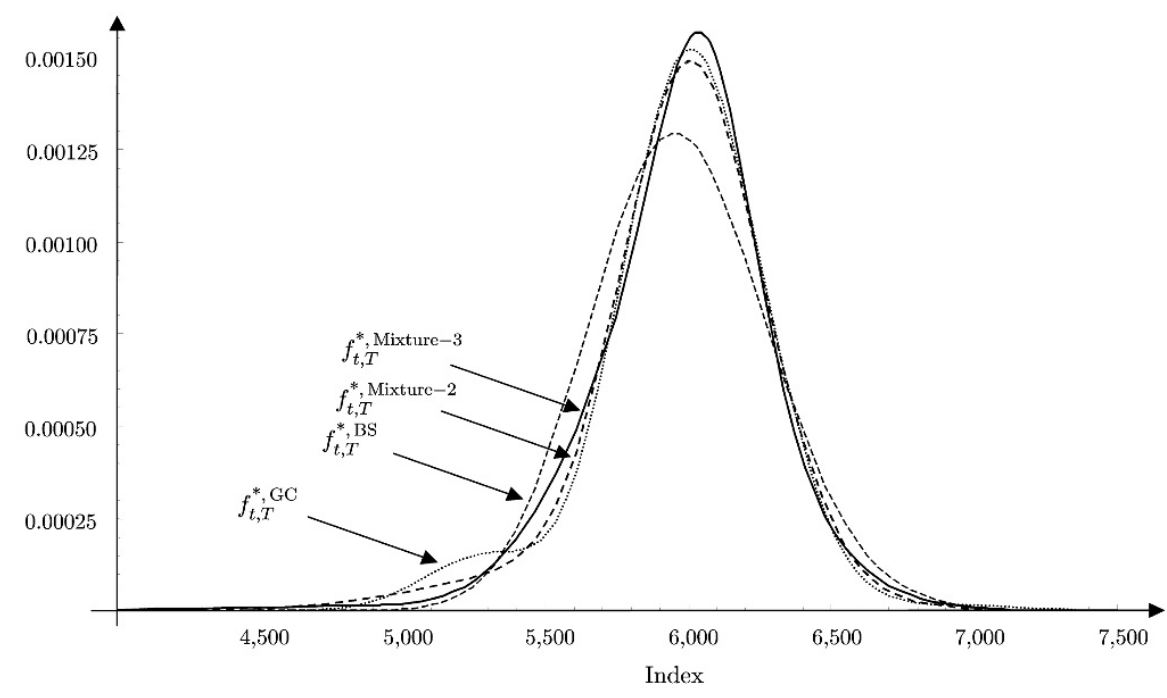

\begin{tabular}{lrrrr}
\hline \hline & \multicolumn{1}{c}{ BS } & \multicolumn{1}{c}{ GC } & Mixture-2 & Mixture-3 \\
\hline Expected value & $5,976.50$ & $5,976.50$ & $5,976.50$ & $5,976.50$ \\
Standard dev. & 309.46 & 319.74 & 320.87 & 325.29 \\
Skewness & 0.1555 & -0.4148 & -0.7057 & -0.9402 \\
Kurtosis & 3.0430 & 4.5779 & 5.2152 & 7.2597 \\
Median & $5,968.50$ & $5,997.80$ & $5,997.00$ & $6,001.78$ \\
Modus & $5,952.55$ & $6,008.71$ & $6,007.13$ & $6,034.23$ \\
IQD $^{*}$ & 416.70 & 359.49 & 366.59 & 361.34 \\
\hline \hline
\end{tabular}

* Interquartile distance

forecast. Based on 2,630 implicit

calibrations for each model, the table provides the overall one-day out-of-sample analysis for DAX options. In total, projected values of $1,178,789$ options are compared with their realised market values, leading to the reported statistics for the model-specific mean and mean absolute errors (ME and MAE) as well as for the mean percentage and mean absolute percentage errors (MPE and MAPE) between model values and market prices.
The statistics for the ME show that the direction of deviation (given in points) is the same for all models, decreasing with an increase in distributional flexibility. The figures for the MAE serve as an indicator that the best forecast is achieved by means of the mixture models: compared with BS and BS-ATM, MAE is, on average, reduced by more than 40 per cent (accompanied by a standard deviation that is approximately 30 per cent lower). Though not part of the optimisation 
Table 2: DAX options: Overall out-of-sample performance

\begin{tabular}{|c|c|c|c|c|c|c|c|c|}
\hline \multirow[b]{2}{*}{ Model } & \multicolumn{4}{|c|}{$M E$} & \multicolumn{4}{|c|}{ MAE } \\
\hline & Mean & Std. dev. & Min. & Max. & Mean & Std. dev. & Min. & Max. \\
\hline BS & -1.99 & 21.26 & $-1,205.65$ & 797.64 & 13.10 & 16.87 & 0.00 & $1,205.65$ \\
\hline BS-ATM & -1.51 & 20.86 & $-1,183.99$ & 426.23 & 13.21 & 16.22 & 0.00 & $1,183.99$ \\
\hline GC & -0.04 & 14.41 & $-1,162.59$ & 784.17 & 6.64 & 12.78 & 0.00 & $1,162.59$ \\
\hline Mixture-2 & -0.75 & 13.13 & $-1,160.66$ & 726.52 & 5.83 & 11.79 & 0.00 & $1,160.66$ \\
\hline \multirow[t]{2}{*}{ Mixture-3 } & -0.74 & 13.11 & $-1,160.64$ & 718.47 & 5.80 & 11.78 & 0.00 & $1,160.64$ \\
\hline & \multicolumn{4}{|c|}{ MPE (in \%) } & \multicolumn{4}{|c|}{ MAPE (in \%) } \\
\hline Model & Mean & Std. dev. & Min. & Max. & Mean & Std. dev. & Min. & $\operatorname{Max}$. \\
\hline BS & -3.41 & 48.15 & -100.00 & $7,562.20$ & 28.80 & 38.74 & 0.00 & $7,562.20$ \\
\hline BS-ATM & -3.38 & 48.98 & -100.00 & $7,562.71$ & 29.22 & 39.45 & 0.00 & $7,562.71$ \\
\hline GC & 0.95 & 24.38 & -100.00 & $6,074.09$ & 12.15 & 21.16 & 0.00 & $6,074.09$ \\
\hline Mixture-2 & -1.34 & 18.54 & -99.92 & $5,811.63$ & 10.08 & 15.62 & 0.00 & $5,811.63$ \\
\hline Mixture-3 & -0.61 & 18.44 & -100.00 & $5,808.42$ & 9.81 & 15.62 & 0.00 & $5,808.42$ \\
\hline
\end{tabular}

approach, the relative deviation measures MPE and MAPE yield a similar picture, favouring the mixture models. The MAPE proves that for these models the absolute percentage error is, on average, more than 60 per cent lower than for the BS and BS-ATM case, while more than halving the standard deviation.

Additionally, option-specific characteristics are examined for their explanatory power with respect to the forecast error. Out-of-sample value differences $\hat{V}-V^{\text {Market }}$ are regressed linearly against moneyness $(M)$, time-to-maturity $(\tau)$ and type of option (given by the indicator variable $\zeta$ with 0: call and 1: put)

$$
\begin{aligned}
\hat{V}_{t}-V_{t}^{\text {Market }}= & a_{0}+a_{1} M_{i}+a_{21} \tau_{i}^{\mathrm{c}} \\
& +a_{22} \tau_{i}^{p}+a_{3} \zeta_{i}+\varepsilon_{i}
\end{aligned}
$$

with $\tau^{c}=\tau(1-\zeta)$ and $\tau^{p}=\tau \zeta$, respectively.
Results for the DAX options are given in Table 3. In order to cope with likely heteroscedasticity in the data set, standard errors are adjusted for heteroscedasticity of unknown form according to White. ${ }^{39}$

For the BS model, the option characteristics exhibit a high explanatory power for the forecast error $\left(R_{\mathrm{adj}}^{2} \approx 0.30\right)$. The constant term $a_{0}$ shows a general overvaluation of approximately 2.90 points. Differentiating by calls and puts by means of $\zeta_{i}$ reveals a strong bias in the pricing of both option types $\left(\mathrm{a}_{3} \approx-7.00\right.$ (points)). The hypothesis that moneyness does not influence the forecast error $\left(a_{1}=0\right)$ cannot be rejected. The coefficients for $\tau^{c}$ and $\tau^{p}$ show the opposite 'direction' of the mispricing depending on time-to-maturity: among long-term options, the BS model overvalues calls and undervalues puts. No similar characteristic is found for the 
Table 3: DAX options: Influence of moneyness, time-to-maturity and option type on price forecasting performance

\begin{tabular}{|c|c|c|c|c|c|c|}
\hline Model & $a_{0}$ & $a_{1}$ & $a_{21}$ & $a_{22}$ & $a_{3}$ & $R_{\mathrm{adj}}^{2}$ \\
\hline \multirow[t]{2}{*}{ BS } & 2.8952 & -0.4476 & 0.1047 & -0.1731 & -6.9966 & 0.3116 \\
\hline & $(19.3 \star \star \star)$ & $(-0.3)$ & $(24.0 \star \star \star)$ & $(-32.3 \star \star \star)$ & $(-30.4 \star \star \star)$ & \\
\hline \multirow[t]{2}{*}{ BS-ATM } & 0.8506 & 1.9298 & 0.1649 & -0.1245 & -6.5056 & 0.3464 \\
\hline & $\left(6.2^{\star \star \star}\right)$ & $(1.5)$ & $\left(45.8^{\star \star \star}\right)$ & $\left(-23.6^{\star \star \star}\right)$ & $\left(-28.8^{\star \star \star}\right)$ & \\
\hline \multirow[t]{2}{*}{ GC } & -0.9485 & -20.9903 & 0.0479 & -0.0814 & 0.9655 & 0.0829 \\
\hline & $\left(-7.8^{\star \star \star}\right)$ & $\left(-20.6^{\star \star \star}\right)$ & $\left(13.8^{\star \star \star}\right)$ & $(-17.4 \star \star \star)$ & $(5.1 \star \star \star)$ & \\
\hline \multirow[t]{2}{*}{ Mixture-2 } & -0.4202 & -5.1908 & 0.0092 & -0.0202 & -0.7224 & 0.0092 \\
\hline & $(-4.0 \star \star \star)$ & $(-5.6 \star \star \star)$ & $\left(3.2^{\star \star \star}\right)$ & $(-4.6 \star \star \star)$ & $(-4.1 \star \star \star)$ & \\
\hline \multirow[t]{2}{*}{ Mixture-3 } & -0.4468 & -5.6732 & 0.0092 & -0.0212 & -0.6548 & 0.0094 \\
\hline & $\left(-4.3^{\star \star \star}\right)$ & $(-6.0 \star \star \star)$ & $\left(3.2^{\star \star \star}\right)$ & $(-4.9 \star \star \star)$ & $(-3.7 \star \star \star)$ & \\
\hline
\end{tabular}

Statistics for two-sided coefficient tests for test value zero are given in parentheses; standard errors are adjusted for heteroscedasticity of unknown form according to White. ${ }^{39}$

$\star \star \star(\star \star, \star)$ : significance at $1 \%(5 \%, 10 \%)$ level.

$R_{\text {adj }}^{2}$ denotes the adjusted coefficient of determination.

moneyness coefficient $a_{1}$. As expected, similar results are obtained in the BS-ATM case.

For the GC model, far less of the variance in the regression can be explained by moneyness, time-to-maturity and option type $\left(R_{\text {adj }}^{2} \approx 0.08\right)$. Compared with the BS reference case, moneyness is now found to have a significant influence on the forecast error. Remarkably, the imbalance in the valuation between calls and puts is much less pronounced $\left(\mathrm{a}_{3} \approx-0.97\right.$ (points)).

The regressions for the mixture models prove the usefulness of flexible distributional assumptions in the case of DAX options: with $R_{\text {adj }}^{2} \approx 0.01$, the forecast error cannot be properly explained by option moneyness, time-to-maturity or option type.

Compared with the GC case, the moneyness coefficient $a_{1}$, for example, is much lower in absolute terms, indicating that the bias in the GC model is far less evident within the mixture framework. Both mixture models exhibit a similar performance, calling the appropriateness of a third distribution kernel into question.

Table 4 provides the results of the forecasting performance of the five models for the group of Euro-Bund-Future options. Based on 657 implicit calibrations for each model, the table provides the overall one-day out-of-sample analysis for Euro-Bund-Future options. In total, projected values of 110,108 options are compared with their realised market values, 
Table 4: Euro-Bund-Future options: Overall out-of-sample performance

\begin{tabular}{|c|c|c|c|c|c|c|c|c|}
\hline \multirow[b]{2}{*}{ Model } & \multicolumn{4}{|c|}{$M E$} & \multicolumn{4}{|c|}{$M A E$} \\
\hline & Mean & Std. dev. & Min. & Max. & Mean & Std. dev. & Min. & Max. \\
\hline BS & -0.0058 & 0.0481 & -1.4685 & 1.0310 & 0.0332 & 0.0353 & 0.0000 & 1.4685 \\
\hline BS-ATM & -0.0098 & 0.0479 & -1.5302 & 0.9957 & 0.0335 & 0.0356 & 0.0000 & 1.5302 \\
\hline GC & -0.0038 & 0.0400 & -1.4931 & 0.9980 & 0.0251 & 0.0313 & 0.0000 & 1.4931 \\
\hline Mixture-2 & -0.0051 & 0.0402 & -1.4773 & 0.9961 & 0.0255 & 0.0315 & 0.0000 & 1.4773 \\
\hline \multirow[t]{2}{*}{ Mixture-3 } & -0.0059 & 0.0404 & -1.4633 & 0.9790 & 0.0257 & 0.0317 & 0.0000 & 1.4633 \\
\hline & \multicolumn{4}{|c|}{ MPE (in \%) } & \multicolumn{4}{|c|}{ MAPE (in \%) } \\
\hline Model & Mean & Std. dev. & Min. & Max. & Mean & Std. dev. & Min. & Max. \\
\hline BS & -2.06 & 15.60 & -99.75 & 355.53 & 10.40 & 11.81 & 0.00 & 355.53 \\
\hline BS-ATM & -3.22 & 15.58 & -99.80 & 343.36 & 10.53 & 11.94 & 0.00 & 343.36 \\
\hline GC & -0.54 & 10.39 & -96.73 & 344.14 & 6.95 & 7.73 & 0.00 & 344.14 \\
\hline Mixture-2 & -0.92 & 10.42 & -93.70 & 343.48 & 7.04 & 7.74 & 0.00 & 343.48 \\
\hline Mixture-3 & -1.13 & 10.44 & -93.47 & 337.57 & 7.03 & 7.80 & 0.00 & 337.57 \\
\hline
\end{tabular}

leading to the reported statistics for the model-specific ME and MAE as well as for the MPE and MAPE between model values and market prices.

For all models, deviation between projected values and realised market prices are economically very small. In a similar manner to the case of DAX options, GC and mixture models lead to a reduction in mean mispricing - judging, for example, from the statistics for MAPE, which is as much as approximately 30 per cent lower than in the BS case.

The regression analysis according to equation (16), shown in Table 5, yields only a moderate influence of option moneyness, time-to-maturity and type on the mispricing within the BS and BS-ATM models $\left(R_{\text {adj }}^{2} \approx 0.16\right)$. With the help of the BS alternatives, none of the three variables is found to explain the forecasting error $\left(R_{\mathrm{adj}}^{2} \approx 0.01\right)$. The GC, Mixture-2 and Mixture-3 models all perform very similarly.

\section{Hedging performance}

To investigate the ability of option pricing models to capture dynamic structures and the correct co-movement between option and underlying over time, hedge portfolios, built model-specifically at time $t$ and monitored for a period of length $\Delta t$ are referred to. A delta hedge is constructed as follows: sell an option at time $t$ and simultaneously enter a position in underlying and a risk-free asset. In the following, a positive sign for a variable denotes a cash inflow due to a sale and a negative sign indicates cash flows from the portfolio due to a purchase.

In the case of a call, a sale at time $t$ and 
Table 5: Euro-Bund-Future options: Influence of moneyness, time-to-maturity, and option type on price forecasting performance

\begin{tabular}{|c|c|c|c|c|c|c|}
\hline Model & $a_{0}$ & $a_{1}$ & $a_{21}$ & $a_{22}$ & $a_{3}$ & $R_{a d j}^{2}$ \\
\hline \multirow[t]{2}{*}{ BS } & 0.0125 & 0.5594 & 0.0001 & -0.0002 & -0.0265 & 0.1587 \\
\hline & $\left(10.4^{\star \star \star}\right)$ & $\left(11.1^{\star \star \star}\right)$ & $(3.3 \star \star \star)$ & $(-4.7 \star \star \star)$ & 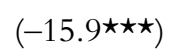 & \\
\hline \multirow[t]{2}{*}{ BS-ATM } & 0.0082 & 0.4999 & 0.0001 & -0.0002 & -0.0265 & 0.1573 \\
\hline & $\left(6.8^{\star \star \star}\right)$ & $(9.9 \star \star \star)$ & $\left(3.2^{\star \star \star}\right)$ & $\left(-5.0^{\star \star \star}\right)$ & $(-15.9 \star \star \star)$ & \\
\hline \multirow[t]{2}{*}{ GC } & 0.0000 & -0.1064 & -0.0001 & -0.0000 & -0.0053 & 0.0033 \\
\hline & $(0.0)$ & $(-2.5 \star \star)$ & $(-2.9 \star \star \star)$ & $(-1.1)$ & $(-3.7 \star \star \star)$ & \\
\hline \multirow[t]{2}{*}{ Mixture-2 } & -0.0011 & -0.0969 & -0.0001 & -0.0000 & -0.0065 & 0.0039 \\
\hline & $(-1.0)$ & $(-2.3 \star \star)$ & $\left(-2.6^{\star \star \star}\right)$ & $(-0.4)$ & $\left(-4.6^{\star \star \star}\right)$ & \\
\hline \multirow[t]{2}{*}{ Mixture-3 } & -0.0031 & -0.1225 & -0.0001 & -0.0000 & -0.0056 & 0.0036 \\
\hline & $\left(-2.8^{\star \star \star}\right)$ & 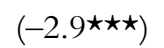 & $\left(-1.8^{\star}\right)$ & $(-0.3)$ & $(-3.9 \star \star \star)$ & \\
\hline
\end{tabular}

Statistics for two-sided coefficient tests for test value zero are given in parentheses, standard errors are adjusted for heteroscedasticity of unknown form according to White..$^{39}$

$\star \star \star(\star \star, \star)$ : significance at $1 \%(5 \%, 10 \%)$ level.

$R_{\text {adj }}^{2}$ denotes the adjusted coefficient of determination.

a simultaneous purchase of $\delta_{t}^{c}$ items of the underlying at current price $S_{t}$ are required. Since cash inflows from the option sale are insufficient to finance this transaction, an additional credit of $B_{t}=\delta_{t}^{c} S_{t}-c_{t}$ is necessary. Analogously, in the case of a put, $\delta_{i}^{\mathrm{p}}$ units of the underlying must be sold.

The cash inflow amounting to $B_{t}=p_{t}+\delta^{\mathrm{p}}{ }_{t} S_{t}$ must be put into a risk-free money market account. The number of units of the underlying, $\delta_{t}^{c}$ and $\delta_{t}^{\mathrm{p}}$, respectively, equals the model-specific sensitivity measure 'delta'

$$
\delta_{t}^{\mathrm{c}}=\frac{\partial c_{t}}{\partial S_{t}} \text { and } \delta_{t}^{\mathrm{p}}=\left|\frac{\partial p_{t}}{\partial S_{t}}\right|
$$

The portfolio value at time $t+\Delta t$ yields the deviation from the reference value zero and measures the model-specific hedge error (HE)

$$
H E_{t+\Delta t}^{c}=-c_{t+\Delta t}+\delta_{t}^{c} S_{t+\Delta t}-\mathrm{e}^{r \Delta t} B_{t}
$$

and

$$
H E_{t+\Delta t}^{\mathrm{p}}=-p_{t+\Delta t}-\delta_{t}^{\mathrm{p}} S_{t+\Delta t}+\mathrm{e}^{r \Delta t} B_{t}
$$

respectively. The smaller the absolute value of HE, the more effectively the model provides risk immunisation. For this specific empirical analysis, average prices of the last three transactions in a certain call or put option on a given day are employed. Hedge errors are calculated over one- and five-trading-day horizons. The model 
Table 6: DAX options: Hedging performance

\begin{tabular}{|c|c|c|c|c|c|c|c|c|}
\hline \multirow[b]{3}{*}{ Model } & \multicolumn{4}{|c|}{ Calls } & \multicolumn{4}{|c|}{ Puts } \\
\hline & \multicolumn{2}{|c|}{ MHE } & \multicolumn{2}{|c|}{ MAHE } & \multicolumn{2}{|c|}{$M H E$} & \multicolumn{2}{|c|}{ MAHE } \\
\hline & Mean & Std. dev. & Mean & Std. dev. & Mean & Std. dev. & Mean & Std. dev. \\
\hline \multicolumn{9}{|c|}{ 1-day interval } \\
\hline BS & 0.39 & 22.09 & 7.48 & 20.78 & 0.35 & 15.43 & 6.10 & 14.18 \\
\hline BS-ATM & 0.34 & 19.17 & 7.43 & 17.67 & 0.35 & 15.43 & 6.09 & 14.18 \\
\hline GC & 0.43 & 22.40 & 7.82 & 21.00 & 0.44 & 15.99 & 6.76 & 14.50 \\
\hline Mixture-2 & 0.49 & 22.78 & 8.19 & 21.26 & 0.50 & 16.22 & 6.92 & 14.68 \\
\hline Mixture-3 & 0.50 & 22.75 & 8.19 & 21.23 & 0.49 & 16.23 & 6.92 & 14.69 \\
\hline \multicolumn{9}{|c|}{ 5-day interval } \\
\hline BS & 1.62 & 32.11 & 18.48 & 26.30 & 2.37 & 26.86 & 15.89 & 21.79 \\
\hline BS-ATM & 1.60 & 30.57 & 18.53 & 24.36 & 2.35 & 26.87 & 15.84 & 21.83 \\
\hline GC & 1.54 & 32.24 & 18.66 & 26.34 & 3.00 & 27.62 & 16.78 & 22.15 \\
\hline Mixture-2 & 1.72 & 32.93 & 19.27 & 26.76 & 3.32 & 28.07 & 17.08 & 22.52 \\
\hline Mixture-3 & 1.73 & 33.03 & 19.31 & 26.85 & 3.33 & 28.03 & 17.07 & 22.49 \\
\hline
\end{tabular}

MHE, mean hedging error; MAHE, mean absolute hedging error.

performance is assessed via the mean hedging error (MHE) and mean absolute hedging error (MAHE) within an interval $[t, t+\Delta t]$.

The results of the hedging analysis for DAX options are documented in Table 6. In total, 29,290 $(26,620)$ 'pairs' of calls and 29,219 (26,091) 'pairs' of puts are built for a one-day (five-day) horizon. For call hedge portfolios with a one-day horizon, the average mean hedge error amounts to 0.39 index points, according to the BS model. The average deviation is slightly lower (0.34) for the BS-ATM model. Remarkably, the average mean error increases for all three more complex models (up to 0.50). A similar picture is obtained for the mean absolute hedge error, with average values around 7.00 (index points). Extending the hedge period to five trading days leads to a comparable 'ranking' among the models and an increase in average mean errors by approximately factor 4.0 for calls and even factor 7.0 for puts. Referring to the MAHE, the error increases by a factor of approximately 2.5. Differentiating further according to moneyness and time-to-maturity of the options (not documented here in detail), the results are very similar. With few exceptions - for example, short-term, far out-of-the-money calls - deviations are lowest for the BS and the BS-ATM models.

Table 7 provides the hedging results for 
Table 7: Euro-Bund-Future options: Hedging performance

\begin{tabular}{|c|c|c|c|c|c|c|c|c|}
\hline \multirow[b]{3}{*}{ Model } & \multicolumn{4}{|c|}{ Calls } & \multicolumn{4}{|c|}{ Puts } \\
\hline & \multicolumn{2}{|c|}{ MHE } & \multicolumn{2}{|c|}{ MAHE } & \multicolumn{2}{|c|}{ MHE } & \multicolumn{2}{|c|}{ MAHE } \\
\hline & Mean & Std. dev. & Mean & Std. dev. & Mean & Std. dev. & Mean & Std. dev. \\
\hline \multicolumn{9}{|c|}{ 1-day interval } \\
\hline BS & -0.59 & 5.93 & 3.83 & 4.56 & -0.62 & 5.85 & 3.98 & 4.33 \\
\hline BS-ATM & -0.59 & 5.92 & 3.82 & 4.56 & -0.63 & 5.86 & 3.99 & 4.34 \\
\hline GC & -0.78 & 6.32 & 4.07 & 4.90 & -0.77 & 6.24 & 4.28 & 4.59 \\
\hline Mixture-2 & -0.80 & 6.37 & 4.07 & 4.97 & -0.73 & 6.19 & 4.25 & 4.56 \\
\hline Mixture-3 & -0.78 & 6.38 & 4.08 & 4.97 & -0.75 & 6.22 & 4.28 & 4.58 \\
\hline \multicolumn{9}{|c|}{ 5-day interval } \\
\hline BS & -1.23 & 14.34 & 9.19 & 11.08 & -1.72 & 13.90 & 9.06 & 10.68 \\
\hline BS-ATM & -1.23 & 14.33 & 9.17 & 11.07 & -1.78 & 13.97 & 9.09 & 10.75 \\
\hline GC & -1.95 & 15.73 & 9.83 & 12.43 & -2.04 & 15.35 & 9.94 & 11.87 \\
\hline Mixture-2 & -2.07 & 15.84 & 9.85 & 12.58 & -2.02 & 15.44 & 9.95 & 11.98 \\
\hline Mixture-3 & -2.12 & 15.92 & 9.89 & 12.65 & -2.04 & 15.40 & 9.94 & 11.94 \\
\hline
\end{tabular}

Note: For ease of notation, all displayed values are multiplied by 100 .

MHE, mean hedging error; MAHE, mean absolute hedging error.

Euro-Bund-Future options. Here, there are 1,167 (959) 'pairs' of calls and 1,357 $(1,134)$ 'pairs' of puts for a one-day (five-day) horizon. All findings are very similar to the DAX options; this is also true for the non-documented differentiation between moneyness and time-to-maturity.

\section{SUMMARY AND OUTLOOK}

This paper has considered the question of which distributional assumption allows the most accurate forecasting of option prices and the most accurate static delta hedging strategy. Employing the Black-Scholes model, the Gram-Charlier density expansion model and two models with mixtures of log-normal distributions on Eurex DAX index and Euro-Bund-Future options data from 1999 and 2000, the study showed that, compared with the Black-Scholes reference model, more flexible distributions led, on average, to a significant reduction in absolute and relative deviations in forecasted prices. The mixture models performed better than the Gram-Charlier model, although the difference for two and three mixture kernels proved to be small. Analysing the forecasting of option prices in detail, it was 
found that the more complex models help to reduce systematic biases relating to option type (call vs put), moneyness and time-to-maturity. In general, the use of more advanced distributional assumptions was shown to be of practical use especially for DAX options with a well-pronounced smile structure of implied volatility.

Conversely, for Euro-Bund-Future options, differences in forecasting performance for the five models were of limited economic relevance. For the latter group, one might even suspect an overfitting of the mixture model with three kernels, also leading to rather unstable distributions over time. Although the limitation in allowed skewness and kurtosis of competing RNDs as in the Gram-Charlier model, can generally be overcome with the help of mixture models, the problem of a likely overparameterisation has to be kept in mind. For example, $\mathrm{Li}^{40}$ recommends that: 'A good SPD (state price density, author's note) is feasible with as few as three parameters: one for volatility, one for skewness, and one for kurtosis' (p. 49).

With respect to hedging performance, average absolute hedging errors compared with the Black-Scholes reference case were found to increase with the flexibility of the underlying RND for both groups of options in the majority of cases. Extending the hedging interval from one to five calendar days, average absolute hedging errors across all models approximately doubled or even tripled. As a general assessment, one might agree with the finding of Dumas et al. ${ }^{2}$ that: 'simpler is better' (p. 2104). Against the background of transactions costs occurring in practice, detected market anomalies in any of the models do not seem to reveal economically relevant arbitrage potential.

While the results support recent research on other international options markets (for example, by $\mathrm{Kim}$ and $\mathrm{Kim}^{24}$ ), the evident limitations of the investigated mixture approach suggest the need for further investigation. Based on cross-sectional data at a given date, the static implied distributions do not consider the evolution of RND over time. In order to cope with the empirically non-flat term structure of implied volatilities, it is necessary to use separate estimations of distributions for any future time interval $[t, T]$. A straightforward extension of the mixture concept would be a 'dynamisation' by means of regime-switching models (see, for example, Bollen $^{41}$ ). In a flexible set-up, however, time-varying transition probabilities in inevitable Monte-Carlo simulations render such an approach computationally demanding. Nevertheless, one has to bear in mind that, in practice, implied RND and especially implied volatilities - serve as measures for pricing options relative to their underlyings. Traders are usually not particularly concerned as to whether implied volatility is really an accurate estimator of volatility over the option's lifetime. ${ }^{42}$ Their role as price substitutes rather suggests, for example, a dependence of implied volatilities on supply and demand. ${ }^{43}$ Future studies - especially those referring to distributional rather than structural model assumptions - might consider, in particular, such empirically proven market phenomena. 


\section{Note}

This paper does not represent an official statement of the firm the author is affiliated to.

\section{References}

1 Bakshi, G., Cao, C. and Chen, Z. (1997) 'Empirical Performance of Alternative Option Pricing Models', Journal of Finance, Vol. 52, pp. 2003-2049.

2 Dumas, B., Fleming, J. and Whaley, R. E. (1998) 'Implied Volatility Functions: Empirical Tests', Journal of Finance, Vol. 53, pp. 2059-2106.

3 Mittnik, S. and Rieken, S. (2000) 'Put-call Parity and the Informational Efficiency of the German DAX-index Options Market', International Review of Financial Analysis, Vol. 9, pp. 259-279.

4 Mittnik, S. and Rieken, S. (2000)

'Lower-boundary Violations and Market-Efficiency: Evidence from the German DAX-Index Options Market', Journal of Futures Markets, Vol. 20, pp. 405-424.

5 Black, F. and Scholes, M. (1973) 'The Pricing of Options and Corporate Liabilities', Journal of Political Economy, Vol. 81, pp. 637-654.

6 Eberlein, E., Keller, U. and Prause, K. (1998) 'New Insights into Smile, Mispricing and Value at Risk: The Hyperbolic Model', Journal of Business, Vol. 71, pp. 371-405.

7 Tompkins, R. G. (1999) 'Implied Volatility Surfaces: Uncovering Regularities for Options on Financial Futures', Working Paper, Vienna University of Technology, Vienna, Austria.

8 Hafner, R. and Wallmeier, M. (2001) 'The Dynamics of DAX Implied Volatilities', Quarterly International Journal of Finance, Vol. 1, pp. 1-27.

9 Brunner, B. and Hafner, R. (2003)

'Arbitrage-free Estimation of the Risk-neutral Density from the Implied Volatility Smile', Journal of Computational Finance, Vol. 7, pp. 75-106.

10 Fengler, M. R., Härdle, W. K. and Villa, C. (2003) 'The Dynamics of Implied Volatilities: A Common Principle Components Approach', Review of Derivatives Research, Vol. 6, pp. 179-202.

11 Fengler, M. R. and Wang, Q. (2003) 'Fitting the Smile Revisited: A Least Squares Kernel Estimator for the Implied Volatility Surface', Working Paper, Humboldt-University Berlin, Institute for Econometrics and Statistics, Berlin, Germany.

12 Rachev, S. T. and Mittnik, S. (1999) 'Stable Paretian Models in Finance', John Wiley, New York, NY.
13 Belledin, M. and Schlag, C. (1999) 'An Empirical Comparison of Alternative Stochastic Volatility Models', Working Paper No. 38 (Series: Finance and Accounting), University of Frankfurt am Main, Frankfurt, Germany.

14 McLachlan, G. and Peel, D. (2000) 'Finite Mixture Models', John Wiley, New York, NY.

15 Ritchey, R. J. (1990) 'Call Option Valuation for Discrete Normal Mixtures', Journal of Financial Research, Vol. 13, pp. 285-296.

16 Bahra, B. (1996) 'Probability Distribution of Future Asset Prices Implied by Option Prices', Bank of England Quarterly Bulletin, Vol. 36, pp. 299-311.

17 Bahra, B. (1997) 'Implied Risk-Neutral Probability Density Functions from Option Prices: Theory and Applications', Working Paper No. 66, Bank of England, London, UK.

18 Bahra, B. (1999) 'Implied Risk-Neutral Probability Density Functions from Option Prices: A Central Bank Perspective', in Knight, J. L. and Satchell, S. E. (Eds), 'Forecasting Volatility in the Financial Markets', Butterworth Heinemann, Oxford, UK, pp. 137-167.

19 Melick, W. R. and Thomas, C. P. (1997) 'Recovering an Asset's Implied Probability Density Function from Option Prices: An Application to Crude Oil During the Gulf Crisis', Journal of Financial and Quantitative Analysis, Vol. 32, pp. 91-115.

20 Guo, C. (1998) 'Option Pricing with Heterogeneous Expectations', Financial Review, Vol. 33, pp. 81-92.

21 Söderlind, P. and Svensson, L. (1997) 'New Techniques to Extract Market Expectations from Financial Instruments', Journal of Monetary Economics, Vol. 40, pp. 383-429.

22 Gemmill, G. and Saflekos, A. (2000) 'How Useful are Implied Distributions? Evidence from Stock Index Options', Journal of Derivatives, Vol. 7, Spring, pp. 83-98.

23 Schittenkopf, C. and Dorffner, G. (2001) 'Risk-neutral Density Extraction from Option Prices: Improved Pricing with Mixture Density Networks', IEEE Transactions on Neural Networks, Vol. 12, pp. 716-725.

24 Kim, I. J. and Kim, S. (2003) 'On the Usefulness of Implied Risk-neutral Distributions Evidence from the Korean KOSPI 200 Index Options Market', Journal of Risk, Vol. 6, Fall, pp. 93-110.

25 Rebonato, R. and Cardoso, M. T. (2004) 'Unconstrained Fitting of Implied Volatility 
Surfaces using a Mixture of Normals', Journal of Risk, Vol. 7, pp. 55-74.

26 Brigo, D. and Mercurio, F. (2002)

'Lognormal-mixture Dynamics and Calibration to Market Volatility Smiles', International Journal of

Theoretical \& Applied Finance, Vol. 5, pp. 427-446.

27 Corrado, C. J. and Su, T. (1996) 'Skewness and

Kurtosis in S\&P500 Index Returns Implied by

Option Prices', Journal of Financial Research,

Vol. 19, pp. 175-192.

28 Cox, J. C. and Ross, S. A. (1976) 'The Valuation of Options for Alternative Stochastic Processes', Journal of Financial Economics, Vol. 3, pp. 145-166.

29 Breeden, D. T. and Litzenberger, R. H. (1978)

'Prices of State-contingent Claims Implicit in Options Prices', Journal of Business, Vol. 51, pp. 621-651.

30 Jackwerth, J. C. (1999) 'Option-implied Risk-neutral Distributions and Implied Binomial Trees: A Literature Review', Journal of Derivatives, Vol. 7, pp. 66-82.

31 Brown, C. A. and Robinson, D. M. (2002) 'Skewness and Kurtosis Implied by Option Prices: A Correction', Journal of Financial Research, Vol. 25, pp. 279-282.

32 Jondeau, E. and Rockinger, M. (2001) 'Gram-Charlier densities', Journal of Economic Dynamics and Control, Vol. 25, pp. 1457-1483.

33 Eurex (2004) 'Products', Frankfurt am Main, Germany, March.

34 Asay, M. R. (1982) 'A Note on the Design of Commodity Options Contracts', Journal of Futures Markets, Vol. 2, pp. 1-7.

35 Lieu, D. (1990) 'Option Pricing with
Futures-style Margining', Journal of Futures Markets, Vol. 10, pp. 327-338.

36 Locatelli, M. (2002) 'Simulated Annealing Algorithms for Continuous Global Optimization', in Pardalos, P. M. and Romeijn, H. E. (eds), 'Handbook of Global Optimization', Vol. 2, Kluwer, Dordrecht, The Netherlands, pp. 179-229.

37 Cooper, N. (1999) 'Testing Techniques for Estimating Implied RNDs from the Prices of European and American-Style Options', Working Paper, Bank of England, London, UK.

38 Bliss, R. R. and Panigirtzoglou, N. (2002)

'Testing the stability of Implied Probability Density Functions', Journal of Banking \& Finance, Vol. 26, pp. 381-422.

39 White, H. L. (1980) 'A Heteroskedasticity-consistent Covariance Matrix Estimator and a Direct Test for Heteroskedasticity', Econometrica, Vol. 48, pp. 817-838.

40 Li, F. (2000) 'Option Pricing: How Flexible Should the SPD Be?', Journal of Derivatives, Vol. 7, pp. 49-63.

41 Bollen, N. P. B. (1998) 'Valuing Options in Regime-switching Models', Journal of Derivatives, Vol. 6, pp. 38-49.

42 Figlewski, S. (1997) 'Forecasting Volatility', Financial Markets, Institutions \& Instruments, Vol. 6, No. 1, pp. 1-88.

43 Bollen, N. P. B. and Whaley, R. E. (2004) 'Does Net Buying Pressure Affect the Shape of Implied Volatility Functions?', Journal of Finance, Vol. 59, pp. 711-753. 